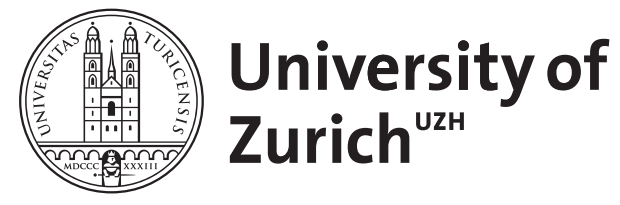

\title{
Current-mode automated quality control cochlear resonator for bird identity
} tagging

\author{
Moeys, D ; Delbruck, T ; Liu, S-C
}

DOI: https://doi.org/10.1109/ISCAS.2015.7168988

Posted at the Zurich Open Repository and Archive, University of Zurich ZORA URL: https://doi.org/10.5167/uzh-121754

Conference or Workshop Item

Accepted Version

Originally published at:

Moeys, D; Delbruck, T; Liu, S-C (2015). Current-mode automated quality control cochlear resonator for bird identity tagging. In: IEEE International Symposium on Circuits and Systems (ISCAS) 2015, Lisbon, 24 May 2015 - 27 May 2015. IEEE Int. Symposium on Circuits and Systems (ISCAS), 1734 1737 .

DOI: https://doi.org/10.1109/ISCAS.2015.7168988 


\title{
Current-Mode Automated Quality Control Cochlear Resonator for Bird Identity Tagging
}

\author{
Diederik Paul Moeys, Tobias Delbrück, and Shih-Chii Liu \\ Institute of Neuroinformatics \\ University of Zürich and ETH Zürich
}

\begin{abstract}
This paper describes a VLSI automatic quality control pitch detector circuit which can be used for detecting the identity of a unique bird. The detector is based on a previous VLSI model of the local gain control mechanism of the outer hair cells of the biological cochlea. This work presents characterization results from a 20-channel chip fabricated in a 4-metal 2-poly CMOS $0.35 \mu \mathrm{m}$ technology with estimated dynamic range of $\mathbf{7 0}$ $\mathrm{dB}$, power consumption of $825 \mathrm{nW}$ per channel, frequency range covering 0.4-10 kHz and mean $Q$ of 6.31. Results are shown for a pitch detection experiment with a tuned resonator.
\end{abstract}

\section{INTRODUCTION}

The local gain control mechanisms implemented in silicon cochleas so far include various feedback schemes to control the gain of a filter based on the input amplitude [1][2]. This work describes experimental results from a modified Automatic Quality Control (AQC) silicon cochlea previously described by Hamilton et al, 2007 [3]. In that reference, only simulations were shown. The authors subsequently demonstrated a working silicon chip which uses a different AQC mechanism with two amplitude settings. The modified AQC circuits of [3] are used here as a basis for a pitch detector circuit. Sections II and III describe the AQC circuit architecture and the chip layout of our circuit. Section IV describes characterization results from the fabricated circuit and Section V describes experimental results from the detection of zebra finches using this pitch detector.

\section{THE CIRCUIT MODEL}

\section{A. Tau-Cell bandpass filter}

The design of the AQC resonator is based on the Tau-Cell bandpass filter (BPF) described in [3] (Fig. 1). The transfer function of this circuit approximates the Hopf equation which has been used to model the local gain control in biological cochleas. A Hopf system is highly sensitive to the input amplitude and frequency near its bifurcation point. Since beyond the bifurcation point the system self-oscillates, it needs, in our case, to operate in the sub-critical stable region. The cochlear resonator consists of two cascaded first-order translinear lowpass filters (LPF), and its output is given by the subtraction between the corresponding output currents of the LPFs. Additional AQC control currents (proportional to a parameter $A$, later explained, and shown flowing into node $\mathrm{V}_{\text {cap } 1}$ in Fig. 1), control the gain of the filter and are generated through a feedback loop (highlighted in Fig. 2). The values of these currents are chosen so that the transfer function of the filter in (1) is an approximation to the Hopf equation:

$$
H(s)=\frac{I_{\text {out }}(s)}{I_{\text {in }}(s)}=\frac{I_{1}(s)-I_{2}(s)}{I_{\text {in }}(s)}=\frac{s \tau}{s^{2} \tau^{2}+(2-A) s \tau+1}
$$

where $\tau=\frac{1}{2 \pi f_{C}}=\frac{C U_{T}}{I_{\tau}}$ is the circuit's time constant, $A$ is an input-dependent control parameter, and $I_{1}$ and $I_{2}$ are the output currents of the first and second stage respectively (Fig. 1). Other variables include $f_{c}$, the resonant center frequency; $C$, the capacitance of each Tau-cell stage; $U_{T}$, the thermal voltage; and $I_{\tau}$, the bias current of the filter. If $A$ is mathematically set to $2-I_{\text {out }}{ }^{2}$, ignoring units, the Hopf equation is wellapproximated.

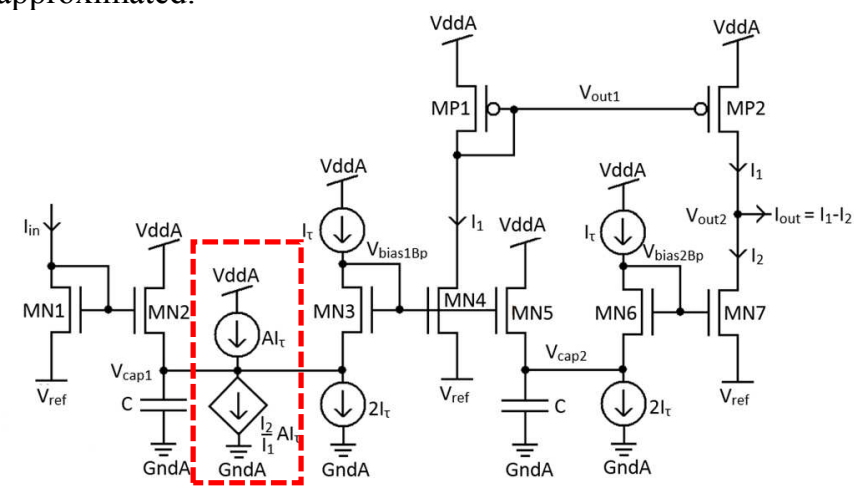

Fig. 1. Translinear bandpass filter with AQC.

The quality factor $Q$ of the BPF in Fig. 1 as described by (1) is dependent on $A$. It grows to infinity as $A$ approaches 2 :

$$
Q=\frac{1}{2-A}
$$

As can be seen from the frequency response of the filter in (3), the denominator simplifies to $\sqrt{2-A}$ when $f=f_{c}$. As a function of the parameter $A$, the gain $(|H(f)|)$ is now approximately hyperbolic with an asymptote at 2 .

$$
|H(f)|=\left|\frac{I_{\text {out }}(f)}{I_{\text {in }}(f)}\right|=\left|\frac{I_{1}(f)-I_{2}(f)}{I_{\text {in }}(f)}\right|=\frac{2 \pi f \tau}{\sqrt{(2 \pi f)^{4} \tau^{4}-A(2 \pi f)^{2} \tau^{2}+1}}(3)
$$

\section{B. The feedback loop}

A Wide Linear Range (WLR) transconductance amplifier based on [4], is used to convert the input voltage into a subthreshold current for the BPF in Fig. 1. The same circuit is also used to convert the output current into a voltage. Using the same dimension transistors in both the WLR amplifiers at the input and output of the AQC circuit allows us to compute the current gain. 
The rest of the current-mode AQC circuit consists of a series of translinear circuits to create the required control currents highlighted in Fig. 1. These currents allow the BPF's original $Q$ of 0.5 to be modified following (2), therefore turning the BPF filter's transfer function into (1). The net control current into $\mathrm{V}_{\text {cap } 1}$ is:

$$
A I_{\tau}-\frac{I_{2}}{I_{1}} A I_{\tau}
$$

where $A$ is nonlinearly dependent on the input amplitude. An overview of the current-mode AQC blocks is shown in Fig. 2.

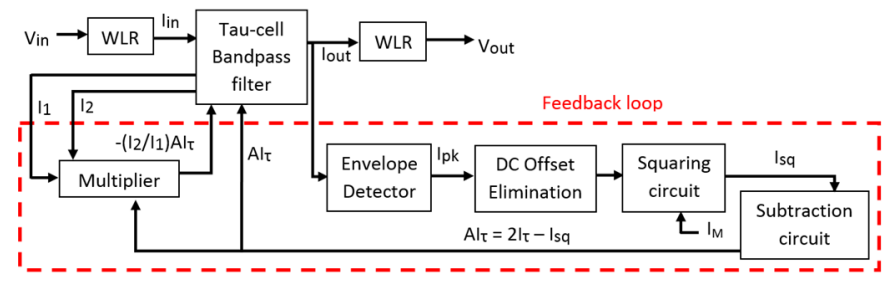

Fig. 2. Overall block diagram of the AQC resonator.

The circuit blocks in Fig. 2 follow approximately those of [3], except that the full-wave rectifier (FWR) block in [3] and the equivalent peak detector block proposed in the improved circuit version of [5] are now replaced by an envelope detector (ED) driven by $I_{\text {out }}$. The authors in [3] had replaced the FWR with a peak detector circuit in [5] but the time constant of the latter circuit, in simulation, proved difficult to be tuned through the nFET leak. When this leak current is set to pico-Amperes, the output of the peak detector which drives the gate of the nFET in the next stage becomes close to Vdd, resulting in an output current with a large de level which reduces the operating range of the next stage. The FWR of [3] was thus chosen initially to be the rectifying part of the ED during our design phase, but the circuit proved to be unnecessary during the testing for the AQC to work and is hence unused (thus, it is omitted in the block diagram). The half-wave rectification part of the ED is in fact found to be well-approximated by the asymmetric negative signal swing caused by the diode connections of the nFETs inside the BPF. A Tau-Cell is then also used as the LPF part of the ED. The resulting output of this stage, $I_{p k}$ is hence a mean current which is proportional to the input current's amplitude and extracted by setting the time constant of the LPF to be much lower than that of the Tau-Cell BPF filter.

The output of this ED LPF however, for no applied ac input signal, still has a dc offset and, therefore, a bias leak transistor in the dc offset elimination block is used to remove this offset along with the accumulated offsets of the previous stages. Without this transistor, the AQC of the fabricated circuit does not work because the large dc current saturates the following stages.

The squaring circuit in Fig. 2 is a simple translinear squarer as used in [3] that computes the square of the peak-detected current $I_{p k}$ and divides it by a set bias current $I_{M}$. The resulting squaring operation gives a current $I_{s q}$ given by (5) for $I_{p k}$ in the order of tens of nano-Amperes at the most:

$$
I_{s q}=\frac{I_{p k}^{2}}{I_{M}}
$$

For a larger $I_{p k}$, the dc elimination transistor of the previous stage can be used to rescale this current. The resulting current in (5) is then subtracted from $2 I_{\tau}$ to obtain $A I_{\tau}$ (and therefore $A$ ), the first term of (4). If the value of $I_{M}$ is chosen to be:

$$
I_{M}=\frac{I_{m}^{2}}{2 I_{\tau}}
$$

where $I_{m}$ is a fixed variable of similar magnitude to $I_{p k}$, then $I_{s q}$ will be of similar magnitude to $2 I_{\tau}$ as shown in (7). The maximum $Q$ for a given current can be set through $I_{M}$. In [3], $I_{M}$ was derived using another squaring circuit, but in our circuit, $I_{M}$ was directly set using a single transistor. This constant $I_{M}$ is suitable in this case because $I_{\tau}$ is fixed during operation. The $A I_{\tau}$ resulting from the subtraction circuit is then given by:

$$
A I_{\tau}=2 I_{\tau}-\frac{I_{p k}^{2}}{I_{m}^{2}} 2 I_{\tau}=2 I_{\tau}-I_{s q}
$$

Finally, to achieve the second term of (4), as in [3], the Enz Punzenberger translinear multiplier is used. This circuit adjusts its bias current dynamically depending on the input current magnitude and has therefore a nonlinear time constant. The problem with this circuit is that for small values of $I_{\tau}$, the transistors of the multiplier can go out of saturation, therefore invalidating the assumption needed for the translinear principle. Our simulations show that bias currents lower than $500 \mathrm{pA}$ result in an output current $I_{2} / I_{1} A I_{\tau}$ that cannot decrease below a certain value (between $500 \mathrm{pA}$ and a few nano-Amperes) depending on the input amplitudes of $I_{1}$ and $I_{2}$ and on the size of the transistors. The result of this problem is an effective reduction of the overall control current of (4) (the second term of (4) does not decrease as much as the first term) and effectively reducing the gain in (3). To mitigate this unwanted decrease in gain (and $Q$ ), AQC circuits with different capacitor values were designed, so as to allow the AQC resonator to cover different center frequencies $(\mathrm{CF})$ using the same $I_{\tau}$.

\section{LAYOUT}

An array of 20 AQC resonators was fabricated in a 4-metal 2-poly $0.35 \mu \mathrm{m}$ CMOS process.

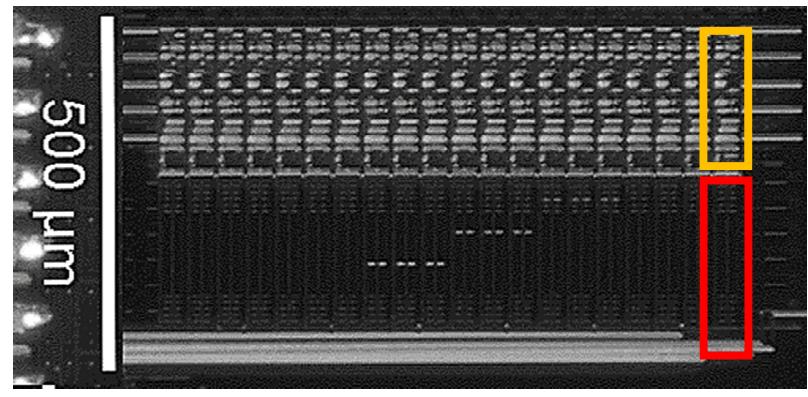

Fig. 3. Microphotograph of the array of AQC cochlear resonators (the rightmost filter's capacitors are highlighted in red and the circuit in yellow).

The two border resonators in Fig. 3 are used as dummies and the other 18 are divided in triplets with MOS capacitors of 1, 2, 4, 6, 8 and $10 \mathrm{pF}$. Every resonator with the same capacitor value has a different $I_{\tau}$ bias, so as to cover a big part of the desired 
frequency range. Each filter has 71 transistors with $\mathrm{W} / \mathrm{L}$ of 4 $\mu \mathrm{m} / 6 \mu \mathrm{m}$ and shares the same $60 \mu \mathrm{m}$ x $60 \mu \mathrm{m}$ WLR input. Every set of 5 resonators is multiplexed to a single output WLR transconductance amplifier.

\section{RESUlts}

\section{A. AQC compression}

The $25 \mathrm{~dB}$ change in the filter gain across different input amplitudes is shown in the left plot of Fig. 4. The fits (dashed lines) are obtained using (3). As predicted, both the filter's $Q$ of (2) and CF decrease with increasing input amplitude (Fig. 4).
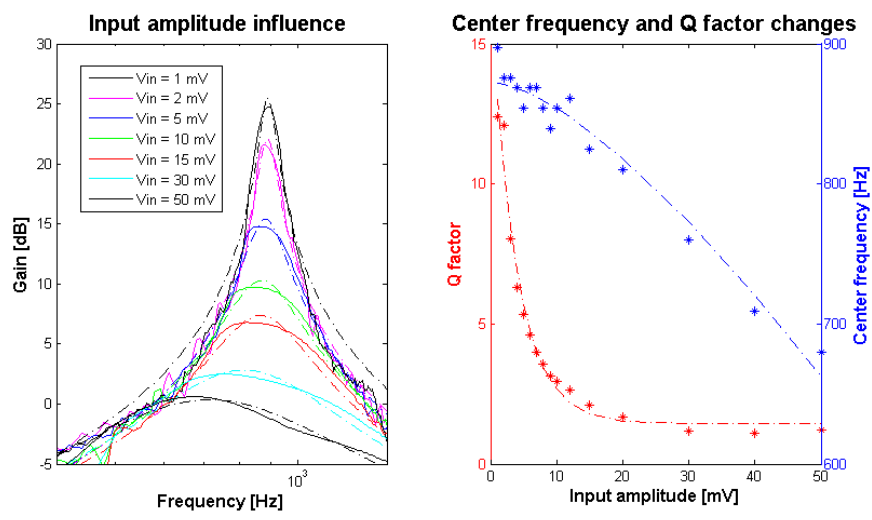

Fig. 4. Left: measured gain and fits (dashed lines) as a function of frequency of a single filter for different input amplitudes. Right: corresponding CF shift and $Q$ change as a function of input amplitude.

\section{B. Frequency tuning and initial gain setting}

For a fixed $5 \mathrm{mV}$ input amplitude, the bias voltage which sets $I_{\tau}$, and therefore the CF, was then swept. A few milli-Volt change in this bias affects the $Q$ of the filter considerably because of the previously mentioned limitation of the EnzPunzenberger multiplier. Decreasing the bias current $I_{\tau}$ has the same effect on $Q$ and $\mathrm{CF}$ of the filter as increasing the input current's amplitude. Even changing $I_{M}$ of the previous squaring block cannot compensate enough for the gain loss. Changes of a few milli-Volts in Vdd also affect the bias current leading to the same effect: a stable supply is therefore very important.

To test if it was possible to set the gain through $I_{M}$ for a fixed input amplitude, this current was swept for a fixed $I_{\tau}$. The gain was found to increase according to (3) and (7), with increasing $I_{M}$. The curves are not shown for lack of space.

\section{Overall channel characteristics and mismatches}

For a fixed $I_{\tau}$ common to all channels, each filter's CF should be determined only by the capacitor's value but this is only roughly the case. In Fig. 5, the maximum gain for every channel is obtained by finding the best bias current for each stage, which turns out to differ by a couple of milli-Volts per filter. Filters with different $I_{\tau}$ to match their highest $Q$ region can still operate in parallel, as there are three different $I_{\tau}$ bias lines wired to every resonator that has the same capacitor.
Transfer functions of resonators plotted with the same colors in Fig. 5 are supposedly identical but because of circuit mismatch, they differ in gain, $Q$ and $\mathrm{CF}$. The $\mathrm{CFs}$ are roughly proportional to the BPF's estimated capacitance. Furthermore, the low-frequency gain of each filter (as in the dashed fits), also varies across filter circuits because of mismatch in the pFET pair MP1 and MP2 mirroring $I_{1}$ on $I_{2}$ (Fig.1).
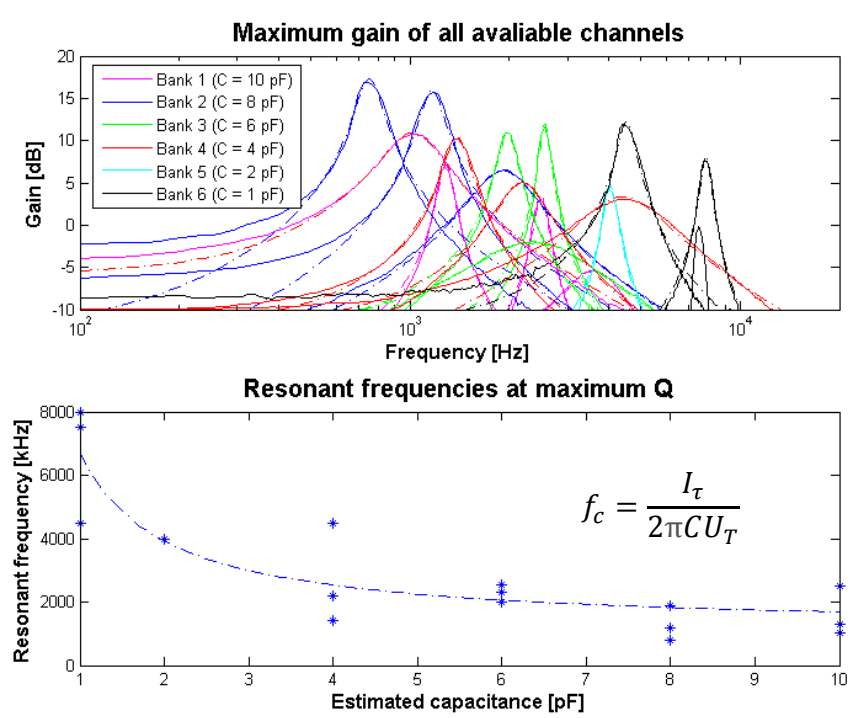

Fig. 5. Top: gain of all available channels for an input voltage of $5 \mathrm{mV}$ and bias current set to individually match the maximum gain operating point. Bottom: the CF scales approximately with capacitance value.

\section{Discussion and specification table}

TABLE I. SPECIFICATION TABLE OF THE AQC RESONATOR

\begin{tabular}{|l|c|}
\hline \multicolumn{2}{|c|}{ AQC specifications } \\
\hline Process & 4 metals 2 poly $0.35 \mu \mathrm{m}$ \\
\hline AQC resonator size & $40.4 \mu \mathrm{m} \times 417.5 \mu \mathrm{m}$ \\
\hline Channels & $\begin{array}{c}20: 3 \mathrm{x}(1 \mathrm{pF}, 2 \mathrm{pF}, 4 \mathrm{pF}, 6 \\
\mathrm{pF}, 8 \mathrm{pF}) \text { and } 5 \mathrm{x} 10 \mathrm{pF}\end{array}$ \\
\hline $\begin{array}{l}\text { Threshold voltage variation for 4/6 } \\
\text { transistor size (using Pelgrom's rule) }\end{array}$ & $\sigma\left(V_{t h}\right)=1.429 \mathrm{mV}$ \\
\hline $\begin{array}{l}\text { Dynamic range (estimated from WLR } \\
\text { input current) }\end{array}$ & $70 \mathrm{~dB}$ \\
\hline Power consumption (from simulations) & $825 \mathrm{nW} / \mathrm{AQC}-$ filter $(250 \mathrm{nA})$ \\
\hline Frequency range & $0.4-10 \mathrm{kHz}$ \\
\hline Maximum $Q$ for ac input of $5 \mathrm{mV}$ & $\mathrm{Mean} 6.31, \mathrm{std} 4.17$ \\
\hline Settling time & $1-6 \mathrm{~ms}$ \\
\hline Maximum input voltage for 5\% THD & $\begin{array}{c}30 \mathrm{mV}(Q=1.3), 5 \mathrm{mV}(Q= \\
2.93)\end{array}$ \\
\hline
\end{tabular}

Overall, it is clear that mismatch is the greatest factor that affects the performance of the chip. Nonetheless, because of the possibility of using 3 different bias currents for every capacitor bank, the $0.4-10 \mathrm{kHz}$ frequency range can be covered with $Q \mathrm{~s}$ of at least 1.5 , partially mitigating this problem. Because of the non-ideality of the multiplier and of general mismatch however, the gain and the $\mathrm{Q}$ factor of every channel cannot be set to be identical. As for the linearity specifications, to increase the 
range of input voltages for low THD, harmonic components can be attenuated by the use of a further BPF with gain, since gain compression and preliminary filtering have been already achieved.

\section{APPLICATION}

The application of this cochlear resonator is demonstrated in a neuroscience setting; in this case, the detection of a unique bird (zebra finch) while singing. The songs of zebra finches in one of our institute's lab are recorded and then converted offline to spectrograms. Each spectrogram is further processed to remove high-frequency noise and fed to a perceptron which was previously trained to detect the pitch (fundamental frequency) of the bird. The pitch of a particular bird is difficult to isolate since it is several decibels lower than its harmonics.

Fig. 6 shows three input spectrograms (left) and the corresponding outputs of the VLSI pitch detector (right). In the first row, interleaving non-overlapping syllables from two different birds are used. Both pitches are equal in amplitude (-60 dBV, therefore barely visible): the target bird (red) has a pitch of $960 \mathrm{~Hz}$ and the non-target bird (black) has a pitch of $700 \mathrm{~Hz}$. The response of the VLSI cochlear resonator, tuned to $960 \mathrm{~Hz} \mathrm{CF}$, shows an attenuation of $10 \mathrm{dBV}$ of all out-of-band frequency components and a $22 \mathrm{dBV}$ boost in the target pitch, now well noticeable (circled in red). The yellow line that spans across the whole output spectrogram at $960 \mathrm{~Hz}$ corresponds to amplified noise in this band. A large $3840 \mathrm{~Hz}$ harmonic in the target bird's song is still visible at the output (circled in dashed black), even though now $7 \mathrm{dBV}$ below the $-38 \mathrm{dBV}$ pitch. Further filtering can be applied to remove higher frequency components (which require more attenuation than $10 \mathrm{~dB}$ ) as zebra finch pitches do not exceed $1.5 \mathrm{kHz}$. A comparator can then be used to tag the identity of the bird.

In the second row, the songs of both birds now overlap but still at the same pitch amplitude. The detector still detects the desired pitch but because of more interferers triggering gain compression, the target signal is boosted to $-45 \mathrm{dBV}$ by a $15 \mathrm{~dB}$ gain. All other signals are still attenuated by $10 \mathrm{dBV}$. The bird's identity can still be tagged even if it is in the presence of other singing birds.

In the third row, the target bird's song is mixed with the song of the second bird but at half the pitch amplitude, to mimic the case when the target bird is further away from the microphone. The response spectrogram of the VLSI detector still allows one to distinguish the target pitch, now at $-49 \mathrm{dBV}$ and boosted by only $14 \mathrm{dBV}$.

\section{CONCLUSION}

The outputs of the fabricated AQC filters fit the theoretical circuit equations, with an average $Q$ factor of 6.31 and $25 \mathrm{~dB}$ gain compression. The outputs are however strongly affected by transistor mismatch as observed in the variation in $\mathrm{CF}$ between identical filters. Further design improvements should be made to reduce mismatch, such as the use of unit transistors and capacitors to achieve desired sizes, and the unused FWR should be removed. The demonstration with bird songs proved the effectiveness of the cochlear resonator if tuned to the desired frequency, showing however the need for calibration. Nonetheless the pitch of a bird can be recognized even with masking, allowing the bird's identity to be tagged. A PCB integrating the AQC circuit and microphones will be developed in the near future to make the circuit portable.
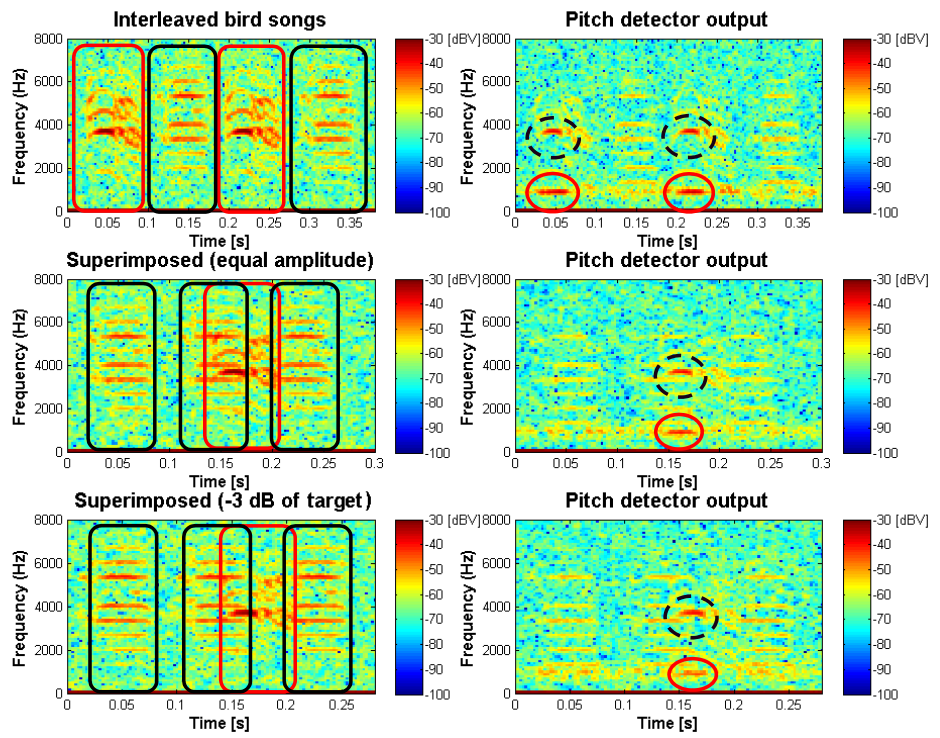

Fig. 6. First row: (left) zebra finch interleaved syllables spectrogram (target in red and non target in black) and (right) corresponding response highlighting detected pitch (red) and out-of-band interferer (dashed black). Second row: (left) superimposed songs of equal pitch amplitude and (right) detector's response. Third row: (left) superimposed songs with target pitch halved in amplitude and (right) pitch detector's response.

\section{ACKNOWLEDGEMENT}

This work was supported by the European Commission project VISUALISE (FP7-ICT-600954) and the Swiss National Science Foundation \#200020_153565 Fast Separation of Auditory Sounds. We would like to thank the Sensors group at INI; and G. Narula and L. Kolb from the Zebra Finch group.

\section{REFERENCES}

[1] A. G. Katsiamis, E. M. Drakakis, and R. F. Lyon, "A Biomimetic, $4.5 \mu \mathrm{W}$, $120+\mathrm{dB}$, Log-Domain Cochlea Channel With AGC," IEEE Journal of Solid-State Circuits, vol. 44, no. 3, pp. 1006-1022, 2009.

[2] M. W. Baker, and R. Sarpeshkar, "Low-Power Single-Loop and DualLoop AGCs for Bionic Ears", IEEE Journal of Solid-State Circuits, vol. 41, no. 9, pp. 1983-1996, 2006.

[3] T. J. Hamilton, J. Tapson, C. Jin, and A. van Schaik, "A 2-D cochlea with Hopf oscillators", IEEE Biomedical Circuits and Systems Conference, Montreal, Canada, 2007.

[4] R. Sarpeshkar, R. F. Lyon, and C. Mead, "A Low-Power Wide-LinearRange Transconductance Amplifier", Analog Integrated Circuits and Signal Processing, 13, 123-151, 1997.

[5] T. J. Hamilton, C. Jin, A. van Schaik, and J. Tapson, "An Active 2-D Silicon Cochlea", IEEE Transactions on Biomedical Circuits and Systems, vol. 2, pp. 30-43, 2008.

[6] B. Wen and K. Boahen, "A silicon cochlea with active coupling", IEEE Transactions on Biomedical Circuits and Systems, vol. 3, no. 6, pp. 444$455,2009$. 